\title{
Intermediate strain rate behaviour of cancellous bone: Links between microstructural and mechanical properties
}

\author{
Marianne $\operatorname{Prot}^{1, \text { a }}$, Trevor Cloete ${ }^{2}$, Dominique Saletti $^{1,3}$, and Sebastien Laporte ${ }^{1}$ \\ ${ }^{1}$ LBM/Institut de Biomécanique Humaine Georges Charpak, Arts et Métiers ParisTech, 151 Boulevard de l'Hôpital, \\ 75013 Paris, France \\ ${ }^{2}$ Blast Impact and Survivability Research Unit (BISRU), Department of Mechanical Engineering, University of \\ Cape Town (UCT), Private bag X3, Rondebosch 7701, South Africa \\ ${ }^{3}$ Univ. Grenoble Alpes, 3SR, 38000 Grenoble, France
}

\begin{abstract}
Relationships between the micro-architecture description of cancellous bone, obtained from medical imaging, and its mechanical properties can be used to assess the compression fracture risk at high and low strain rate. This study extends the rupture prediction to the intermediate strain rate regime. The micro-architecture description was obtained with a CT-scan, for which geometry, topology, connectivity and anisotropy parameters were computed and compared to mechanical identified parameters in order to confirm their usefulness. Three strain rates were investigated: 1/s, 10/s and 100/s using two different devices: a Wedge-Bar apparatus and a conventional split Hopkinson pressure bar implemented with a Cone-in-Tube striker and a tandem momentum trap. This setup provides a constant strain rate loading with routine specimen recovery allowing the fracture zone to be investigated. This study reveals that a transition in the response behaviour occurred in the intermediate regime and confirms the significant porous organization influence through the regimes.
\end{abstract}

\section{Introduction}

Human musculoskeletal modeling requires accurate knowledge of bone failure response including cancellous bone fracture. Literature studies have focused on extreme loadings (low [1] and recently high [2,3] strain rates). The intermediate strain rate (ISR) regime ( 1 and 10/s) has been characterized [4,5] but the observed scatter was not investigated or linked to the upper ISR limit $(100 / \mathrm{s})$ and extreme strain rates. Detailed features of the micro-architecture of porous cancellous bone [6] have been linked to the quasi-static behaviour, to the dynamic and confined dynamic loading [7] to predict the fracture localization [8], the micro-damage [9] and the global mechanical response [10].

In this study, bovine bone specimens were compressed up to the point of trabeculae collapse, at 3 strain rates in the ISR regime (1/s to $100 / \mathrm{s})$ using two different devices: a Wedge-Bar apparatus [11,12] and a conventional split Hopkinson pressure bar (SHPB) implemented with a Cone-in-Tube (CiT) striker [12] and a tandem momentum trap [13]. Both testing techniques allow for specimen recovery after a single loading event. All the specimens were micro-CT scanned before and after testing to investigate the pre- and post-compression architecture.

The aim of this study is to quantify the links between cancellous architectural descriptors and macroscopic mechanical behaviour including fracture initiation under ISR compression loading.

\footnotetext{
${ }^{a}$ Corresponding author: marianne.prot@ensam.eu
}

\section{Methods}

\subsection{Samples}

42 cylindrical bovine cancellous bone specimen (length: $7.5 \mathrm{~mm}$; diameter: $10.5 \mathrm{~mm}$ ) were slowly cut from 6 different femoral bones in order not to damage the peripheral trabeculae. The anatomical position and orientation of each specimen was recorded (i.e.: femoral head versus great trochanter and perpendicularity with respect to one of 3 anatomic planes: sagittal, transversal, frontal). The non-defatted specimens were stored in a frozen condition in a saline solution. Samples were thawed for 24 hours at $+5^{\circ} \mathrm{C}$ before being brought to room temperature prior to experiments.

\subsection{Pre-compression microstructural properties}

A General Electric phoenix v|tome|x L240/NF180 microCT scanner was used to identify the specimen architecture with a low dose of radiation. X-ray settings were $70 \mathrm{kV}$ and 350 microA, acquisition time of $500 \mathrm{~ms}$ per image, with no averaging and no skipping of images, for a resolution of approximately $80 \mu \mathrm{m}$. The Otsu [14] multi-threshold segmentation technique was used to automatically separate bone from marrow and background. In accordance with recommendations in the literature $[1,15]$, architectural parameters were extracted from 3D and skeletonized images using the codes CTan and BoneJ. The eleven architectural parameters summarized in Table 1, are divided into descriptors of geometry, topology, connectivity and anisotropy. 
Table 1. Summary of architectural parameters.

\begin{tabular}{|l|l|l|}
\hline Parameter & Description & Unit \\
\hline BV/TV & Bone Volume/ Total Volume & $\%$ \\
Tb.Th & Mean thickness of trabeculae & $\mathrm{mm}$ \\
Tb.Sp & Trabecular Separation \\
Conn.D & $\begin{array}{l}\text { Connectivity Density or number of } \\
\text { trabeculae per unit volume }\end{array}$ & $/ \mathrm{mm} 3$ \\
\hline FD & $\begin{array}{l}\text { Fractal Dimension } \\
\text { SMI } \\
\text { Structure Model Index }\end{array}$ & \\
\hline $\begin{array}{l}\text { N.Nd } \\
\text { N.Tp }\end{array}$ & $\begin{array}{l}\text { Number of Junctions } \\
\text { Number of Triple Point, i.e. Number of }\end{array}$ & $/ \mathrm{mm} 3$ \\
N.Qp & $\begin{array}{l}\text { Junctions with 3 branches } \\
\text { Number of Quadruple Point, i.e. Number of }\end{array}$ & $/ \mathrm{mm} 3$ \\
\hline $\begin{array}{l}\text { DA } \\
\text { MIL }\end{array}$ & $\begin{array}{l}\text { Junctions 4 branches } \\
\text { Mean Intercept Length (3 parameters) }\end{array}$ & $\mathrm{mm}$ \\
\hline
\end{tabular}

Table 2. Specimen test repartition.

\begin{tabular}{|c|c|c|c|}
\hline Regime & \multicolumn{3}{|c|}{ Intermediate } \\
\hline Strain Rate & $1 / \mathrm{s}$ & $10 / \mathrm{s}$ & $100 / \mathrm{s}$ \\
\hline Apparatus & Wedge & Wedge & SHPB \\
\hline Number of Specimens & 11 & 13 & 18 \\
\hline
\end{tabular}

\subsection{Experimental technique}

Experiments were performed as described by the authors in a previous study [16]. Two different devices were used. A conventional SHPB system implemented with a CiT striker and a momentum trap system was used to compress specimens at 100/s while a Wedge-Bar apparatus was used to load specimens at $1 / \mathrm{s}$ and $10 / \mathrm{s}$. The recovery method was set in order to stop the loading after $10 \%$ strain. ISR compression tests using the Wedge-Bar were filmed using a Photron Fastcam -APX RS250k at 50000 frames per seconds for a resolution of 128 per 192 pixels $^{2}$.

The samples with observed architectural abnormalities or with too much noise in the signal were discarded from the study. The number of specimen tested at the three strain rates is given in Table 2 .

\subsection{Mechanical properties}

A robust automated procedure was developed to determine the mechanical properties through all the three different strain rates. Inspired by a logistic risk curve [17], the stress strain behaviour was model with a 5 parameters law. A robust non-linear least square method was used to set the apparent Young's Modulus, $\mathrm{E}_{\mathrm{app}}$, as the inflexion point slope, the yield Stress and Strain, $\varepsilon_{\mathrm{y}} \& \sigma_{\mathrm{y}}$, the ultimate Stress and Strain, $\varepsilon_{\mathrm{u}} \& \sigma_{\mathrm{u}}$. In addition, error estimates were obtained using the bootstrap method [18].

\subsection{Data analysis}

A Kruskal-Wallis test was used to assess that there is no difference between the micro-architectural values in the 3 sets of strain rates. A p-value of 0.05 was chosen as the upper threshold of significance. Statistical tests were performed using XLSTAT@ software.

The limited number of specimens dedicated to each strain rate could adversely affects the accuracy of the
Table 3. Mean values and standard deviation (italic) of architectural parameters. Non-exhaustive list.

\begin{tabular}{|c|c|c|}
\cline { 2 - 3 } \multicolumn{1}{c|}{} & BV/TV & Tb.Th \\
\hline $\mathbf{1 / s}$ & $54.8(7)$ & $0,32(0.003)$ \\
\hline $\mathbf{1 0 / s}$ & $51.5(9)$ & $0,32(0.003)$ \\
\hline $\mathbf{1 0 0 / \mathbf { s }}$ & $51.2(14)$ & $0,33(0.003)$ \\
\hline
\end{tabular}

Spearman tests usually used to highlight correlation between architectural and mechanical parameters. The Mann-Whitney statistical test was previously performed to appraise the strain rate grouping into one intermediate regime more trustworthy for cross-correlations.

The mechanical parameters sensitivity to strain rate needs to be carefully considered as 6 different bones and 3 anatomical orientations were used for sampling. A variance analysis was used to qualitatively investigate the relative influence of the bone provenance, the anatomic direction, the location in the femur and the strain rate. The influence quantification was appraised using the KruskalWallis test.

\subsection{Post- compression investigation}

A similar procedure to the pre-compression architecture acquisition was completed after testing. Specimen scanning thresholds were set to the same level as the pre-compression procedure. Pre- and post-compression specimen image registrations were done on Avizo@) Specimen scans were superimposed using a rigid transformation and used to compute a histogram-based correlation between the model and the reference.

\section{Results and discussion}

\subsection{Microstructure properties}

Mean values and standard deviations of microstructure parameters are given in Table 3.

The magnitude of the observed variations is consistent with previously published data on bovine cancellous bone [15].

The difference between architectural parameters at the three strain rates was found to be insignificant by the Krusal-Wallis test. Then the data can be treated as a single dataset.

\subsection{Mechanical properties}

A typical example of stress-strain behaviour with its mechanical parameters identification is presented in Fig. 1.

The method, providing good results and was not biased by noise while effectively dealing with the toe region of the curve. A maximum estimation error was observed at $10 / \mathrm{s}$ due to load cell noise.

The values of mechanical parameters are broadly in agreement with the literature dedicated to bovine femur properties under quasi-static loading [19]. Cancellous bone is known to be strain rate dependent [20]. The results are lower than those for the dynamic characterization of bovine tibia [21] but higher than human tibia results [3]. Results were in accordance with the previous statement 


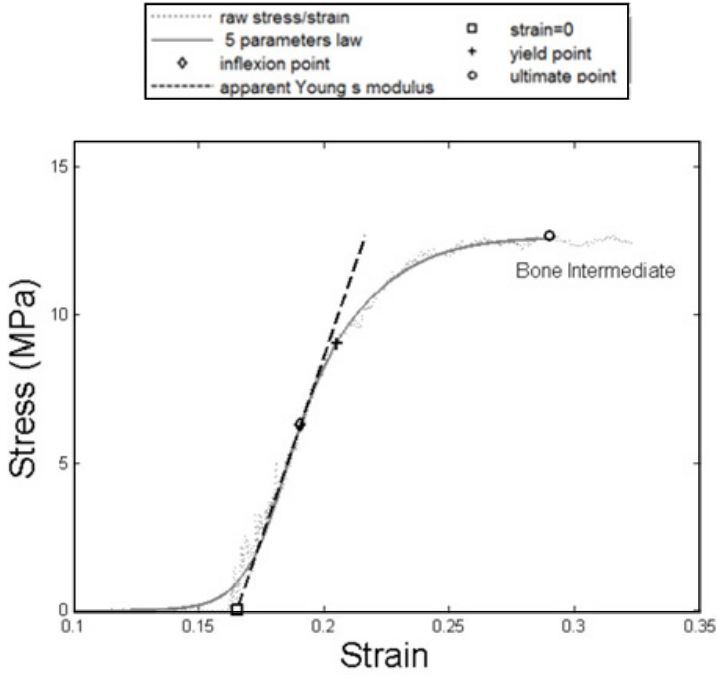

Figure 1. Mechanical parameters determination at 1/s.

regarding $\mathrm{E}_{\mathrm{app}}, \sigma_{\mathrm{y}} \& \sigma_{\mathrm{u}}$ while the yield and ultimate strains appear higher at $1 / \mathrm{s}$. For this specific behaviour, stick and slip mechanism and time effect were discarded thanks to footage and the raw data. Value variability is also higher at 1/s. Further investigations on the marrow effect at intermediate strain rate loadings will be developed in order to explain this difference.

The Mann Whitney test was used to appraise the strain rate grouping. The results for $\varepsilon_{y}$ and $\varepsilon_{u}$ confirm that a distinct transition occurs between the three strain rates. The value of $E_{a p p}$, appears to be similar for $10 / \mathrm{s}$ and $100 / \mathrm{s}$ while results at $1 / \mathrm{s}$ are close to the quasi-static case. Finally the yield and ultimate stress confirmed that all the three strain rates can be considered as one intermediate regime.

\subsection{Sensitivity of the behaviour}

The Fig. 2 presents a non-exhaustive illustration of the ultimate stress sensitivity to the anatomic planes (Fig. 2(a) and the sampling location in the proximal femur (Fig. 2(b).

The variance results confirms that the plane supporting the body weight is presenting a higher $\sigma_{u}$. The higher ultimate stress value from the femoral head location versus the values from the great trochanter location also supports the anatomic hypothesis regarding the real loading [22]. Mechanical parameters were consistent between the six bones with a maximum Kruskal-Wallis p-value of 0.54 for $\sigma_{y}$.

Due to the limited number of specimen tested in regard to all the configurations (bone provenance, femoral head/great trochanter, anatomic plan, strain rates), no cross-interaction could be studied.

Samples extracted from the same bone and adjacent anatomical positions are compared in Fig. 3.

As suggested by the literature focused on quasi-static $[23,24]$ and dynamic strain rates [7], an architecture effect (geometry, connectivity, topology, anisotropy) contributes to the experimental scatter observed in Fig. 3.
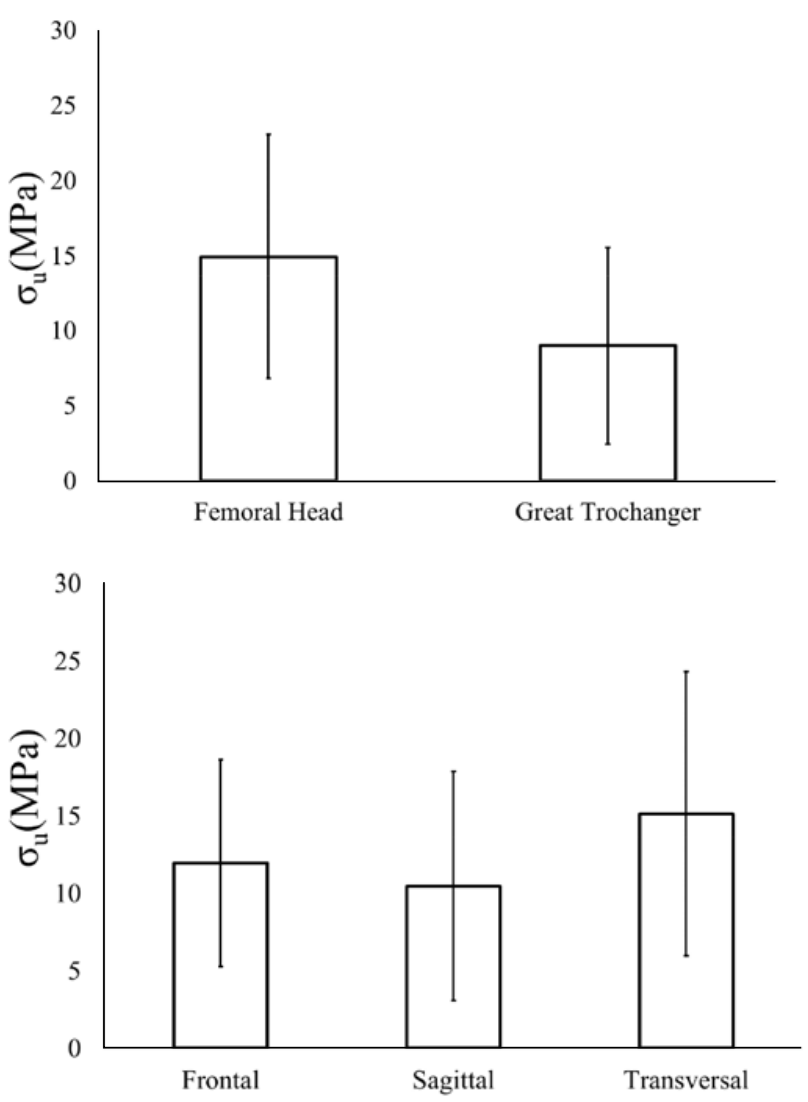

Figure 2. Ultimate stress sensitivity: a) to the anatomic planes, b) to sampling location in the proximal femur.

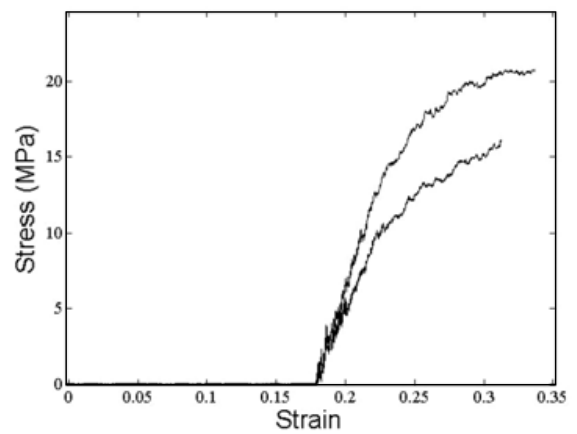

Figure 3. Comparison of two closely extracted specimens at strain rate of $1 / \mathrm{s}$ (raw curve).

Table 3. Mean values and standard deviation (italic) of identified mechanical parameters.

\begin{tabular}{|l|c|c|c|c|c|}
\cline { 2 - 6 } \multicolumn{1}{c|}{} & $\mathbf{E}_{\text {app }}(\mathbf{M P a})$ & $\boldsymbol{\varepsilon}_{\mathbf{y}}(\boldsymbol{\%})$ & $\sigma_{\mathbf{y}}(\mathbf{M P a})$ & $\boldsymbol{\varepsilon}_{\mathbf{u}}(\boldsymbol{\%})$ & $\boldsymbol{\sigma}_{\mathbf{u}}(\mathbf{M P a})$ \\
\hline $\mathbf{1 / s}$ & $286(7)$ & $4(2)$ & $8(5)$ & $11(5)$ & $12(8)$ \\
\hline $\mathbf{1 0} / \mathbf{s}$ & $543(9)$ & $2(1)$ & $10(6)$ & $5(2)$ & $14(8)$ \\
\hline $\mathbf{1 0 0} / \mathbf{s}$ & $629(14)$ & $2(0)$ & $11(7)$ & $3(1)$ & $13(9)$ \\
\hline
\end{tabular}

\subsection{Relationships between parameters}

The micro-architecture description obtained with a CTscan was compared to mechanical identified parameters in order to confirm their usefulness. Spearman test results for combined strain rates are presented in Table 4.

The mechanical parameters presented are linked with geometry and topology architectural parameters such as 
Table 4. Spearman test results. Non-exhaustive list. *: Correlation between the parameters ( $\mathrm{p}$-value $<0.05$ ).

\begin{tabular}{|c|c|c|c|c|c|c|c|}
\hline Variables & BV/TV & Tb.Th & Tb.Sp & Tb.N & Conn.D & SMI & FD \\
\hline $\mathrm{E}_{\text {app }}$ & $*$ & $*$ & & & $*$ & $*$ & $*$ \\
\hline$\sigma_{\mathrm{y}}$ & $*$ & $*$ & $*$ & $*$ & $*$ & $*$ & $*$ \\
\hline$\sigma_{\mathrm{u}}$ & $*$ & $*$ & $*$ & $*$ & $*$ & $*$ & $*$ \\
\hline
\end{tabular}
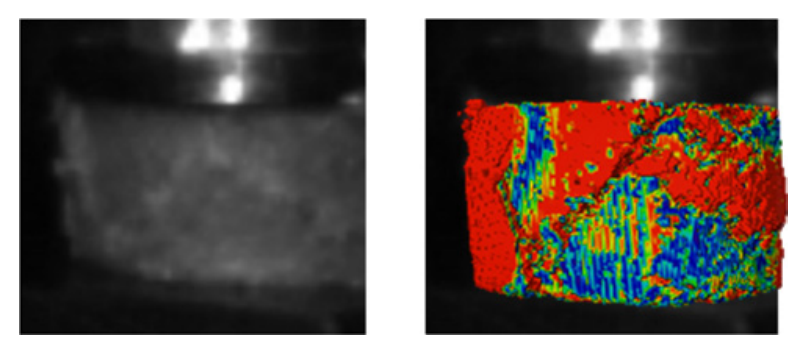

Figure 4. a) Footage at 10/s. b) Distance illustration between the surface of the post and pre- registered compressed reconstructed 3D CT-scans.

the connectivity parameters (see Table 1 ) not presented in this Table 4. However, the anisotropy parameters (3 MILs and DA) are not linked with the behaviour. A projection along the compression axis will overcome the bias inherent in the 3 anatomical plane considered in the study.

Architectural parameter outliers were included in the data set as their effect, while noticeable, was not found to significantly affect the correlations.

The resulting correlations generally concur with the published quasi-static [23,24] and dynamic [7] published study. Trabecular separation, Tb.Sp, was not found to correlate with the Young's Modulus. This result is in contrast with result reported in the literature where such correlation was obtained. The resolution of this issue will require further test using the robust data processing methodology presented in this paper.

\subsection{Post- compression investigation}

Specimens were successfully recovered after a single loading event of a predetermined duration at a strain of approximately $10 \%$. This allows the fracture localization to be investigated as depicted in Fig. 4.

The displacement evaluation of the pre- and postregistered surface extracted from the micro-scans appears to provide a good correlation with the observed fracture zone. Ongoing work to quantifying this fracture behaviour will allow a more precise description that could be implemented in cancellous bone architecture-based model.

\section{Conclusion}

This study presents the relationship between cancellous bone architectural parameters and mechanical response parameters at low and high strain rates in the intermediate strain rate regime (ISR: 1 to 100/s). This work presents a new approach to characterizing the mechanical strength of cancellous bone subjected to intermediate loading rates via architectural parameters descriptions. These results can be implemented in material models for cancellous bone for the prediction of typical dynamic fractures in both the lower to the higher strain rate regimes.

The authors wish to thank Mr. S. Sacks of Sack Butcheries for supplying the bovine femurs, Mr. C. Harris (UCT, Medical School) for the machining of the specimens, Mr. P. Smith and Mr. G. Newins (UCT, Department of Mechanical Engineering) for machining the setup components and the UCT Research Committee for the financial support to conduct this work.

\section{References}

[1] H. Follet, S. Viguet-Carrin, B. Burt-Pichat, et al., J. Orthop. Res., 29(4), 481-488 (2011)

[2] A. Pilcher, W. Wang, Z. Kaltz, et al., J. Biomech. Eng., 132(8) (2010)

[3] C.K. Teja, A. Chawla, S. Mukherjee, Int J Crashworthiness, 18(1), 11-18 (2013).

[4] D.R. Carter, W.C. Hayes, J. Bone Joint Surg. Am, 59, 954-962 (1977)

[5] F. Linde, P. Norgaard, A. Odgaard, et al., J. Biomech., 24(9), 803-809 (1991)

[6] M. Stauber, A. Nazarian, R. Muller, J. Bone Min Res, 29(1), 134-141 (2006)

[7] M. Prot, D. Saletti, S. Pattofatto, V. Bousson, S. Laporte, J. Biomech, 48 (3), 498-503 (2015)

[8] C.J. Hernandez, F.M. Lamber, J. Widjaja, C. Chapa, C.M. Rimnac, Bone, 66, 205-213 (2014)

[9] S. Tassani, G.K. Matsopoulos, J. Biomech., 60, 7886 (2014)

[10] B. Zhou, X. Sherry Liu, J. Wang, et al., J. Biomech., 47(3), 702-708 (2014)

[11] T.J. Cloete, M. Stander, EPJ Web of Conf, 26, 01025 (2012)

[12] T.J. Cloete, G. Paul, E.B. Ismail, Philos Trans A Math Phys Eng Sci, 372 (2015)

[13] M. Prot, T.J. Cloete, S. Pattofatto, EPJ Web of Conf, 26, 03003 (2012)

[14] N. Otsu, Automatica, 11(285-296), 23-27 (1975)

[15] A. Syahrom, MRA. Kadir, J. Abdullah \& A. Ochsner, 49(12), Med. Biol. Eng. \& Comp. 1393-1403 (2011)

[16] M. Prot, T.J. Cloete, D. Saletti, S. Laporte, Comput. Meth. Biomec., 17(S1), 50-51 (2014)

[17] J.H. Ricketts, G.A. Head, Am. J. Phy. 277(2), R441R454 (1999)

[18] B. Efron, R.J. Tibshirani, An introduction to the bootstrap, CRC press, 57 (1994)

[19] V. Kefalas, D.A. Eftaxiopoulos, J. Mech. Behav. Biomed., 6, 41-52 (2012)

[20] R.M. Guedes, J.A. Simoes \& JL. Morais, J. Biomech., 39(1), 49-60 (2006)

[21] A. Pilcher, X. Wang, Z. Kaltz, J.G., Garrison, G.L., Niebur, J., Mason, W. Chen, J. Biomed. Eng. 132(8), 081012 (2010)

[22] P.A. Hulme, S.K. Boyd, S.J. Ferguson, Bone 41(6), 946-957 (2007)

[23] E. Mittra, C. Rubin, Y.X. Qin, J. Biomech. 38(6), 1229-1237 (2005)

[24] J. Teo, K.M. Si-Hoe, J.E.L. Keh, S.H. Teoh, Clin. Biomech., 21(3), 235-244 (2006) 